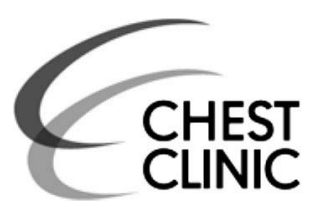

Thoracic Surgery, Renmin Hospital of Wuhan University, Wuhan, Hubei, China

\section{Correspondence to} Professor Qing Geng, Department of Thoracic Surgery, Renmin Hospital of Wuhan University, Wuhan 430060, China; szgqing@126.com

Received 29 January 2018 Revised 14 March 2018 Accepted 19 March 2018 Published Online First 5 April 2018

\title{
'Malignant' pleural effusion with abdominal gossypiboma
}

\author{
Tao Fan, Wei Wang, Xiaobo Guo, Qing Geng
}

\section{DESCRIPTION}

Following a 15-day history of cough with associated breathlessness, a 50-year-old woman presented with bilateral pleural effusions and a pericardial effusion. Her medical history included removal of an appendiceal cyst and a hysteromyoma resection 12 years previously. There was no cardiac or respiratory history. Physical examination revealed decreased breath sounds bilaterally with distant heart sounds heard. Pleural fluid demonstrated high levels of CA125 $(2909.70 \mathrm{U} / \mathrm{mL}$; reference range, 3.6-68.6 U/mL), cytokeratin fragment (CYFRA) $(724.00 \mathrm{ng} / \mathrm{mL}$; range, $0-3.3 \mathrm{ng} / \mathrm{mL})$, squamous cell carcinoma antigen (SCC) $(37.47 \mathrm{ng} / \mathrm{mL}$; range, $0-1.5 \mathrm{ng} / \mathrm{mL}$ ) and neuron specific enolase (NSE) (31.94 ng/mL; range, $0-16.3 \mathrm{ng} / \mathrm{mL}$ ). Serum samples demonstrated high levels of CA125 $(227.60 \mathrm{U} / \mathrm{mL}$; range, 3.6-68.6 U/mL), CYFRA $(6.79 \mathrm{ng} / \mathrm{mL}$; range, $0-3.3 \mathrm{ng} / \mathrm{mL})$, NSE $(23.14 \mathrm{ng} / \mathrm{mL}$; range, $0-16.3 \mathrm{ng}$ / $\mathrm{mL})$ and CA199 $(39.13 \mathrm{U} / \mathrm{mL}$; range, $0-37 \mathrm{U} / \mathrm{mL})$. Serum markers for tuberculosis and rheumatoid arthritis were within the normal range, although C reactive protein $(70.90 \mathrm{mg} / \mathrm{L}$; range, $0-10 \mathrm{mg} / \mathrm{L})$ and erythrocyte sedimentation rate $(35 \mathrm{~mm} /$ hour; range, $0-20 \mathrm{~mm} /$ hour) were elevated. Positron emission tomography-CT (PET-CT) confirmed the presence of a hypermetabolic mass $\left(\mathrm{SUV}_{\max } 10.7\right.$, delay 1 hour, $\mathrm{SUV}_{\max } 14.0$ ) measuring $10.5 \times 7.5 \mathrm{~cm}$ in the abdominal cavity (figure 1 ), which prompted a laparotomy. At laparotomy, a $9.5 \times 7.5 \times 6.3 \mathrm{~cm}$ mass was resected and its cut surface was consistent with a surgical gauze swab (figure 2A). Histopathological examination revealed that the mass was a surgical gauze swab with features of a chronic granuloma (figure 2B). A diagnosis of gossypiboma was definitively confirmed.

The pericardial effusion and pleural effusions were observed to have resolved 1 month following surgery. In addition, tumour marker levels returned to normal, except for NSE $(19.03 \mathrm{ng} / \mathrm{mL})$. At 5 years follow-up after the operation, the patient demonstrated good recovery and absence of any lesions.
Check for updates

To cite: Fan T, Wang W, Guo $X_{\text {, et al. Thorax }}$ 2018:73:992-993.

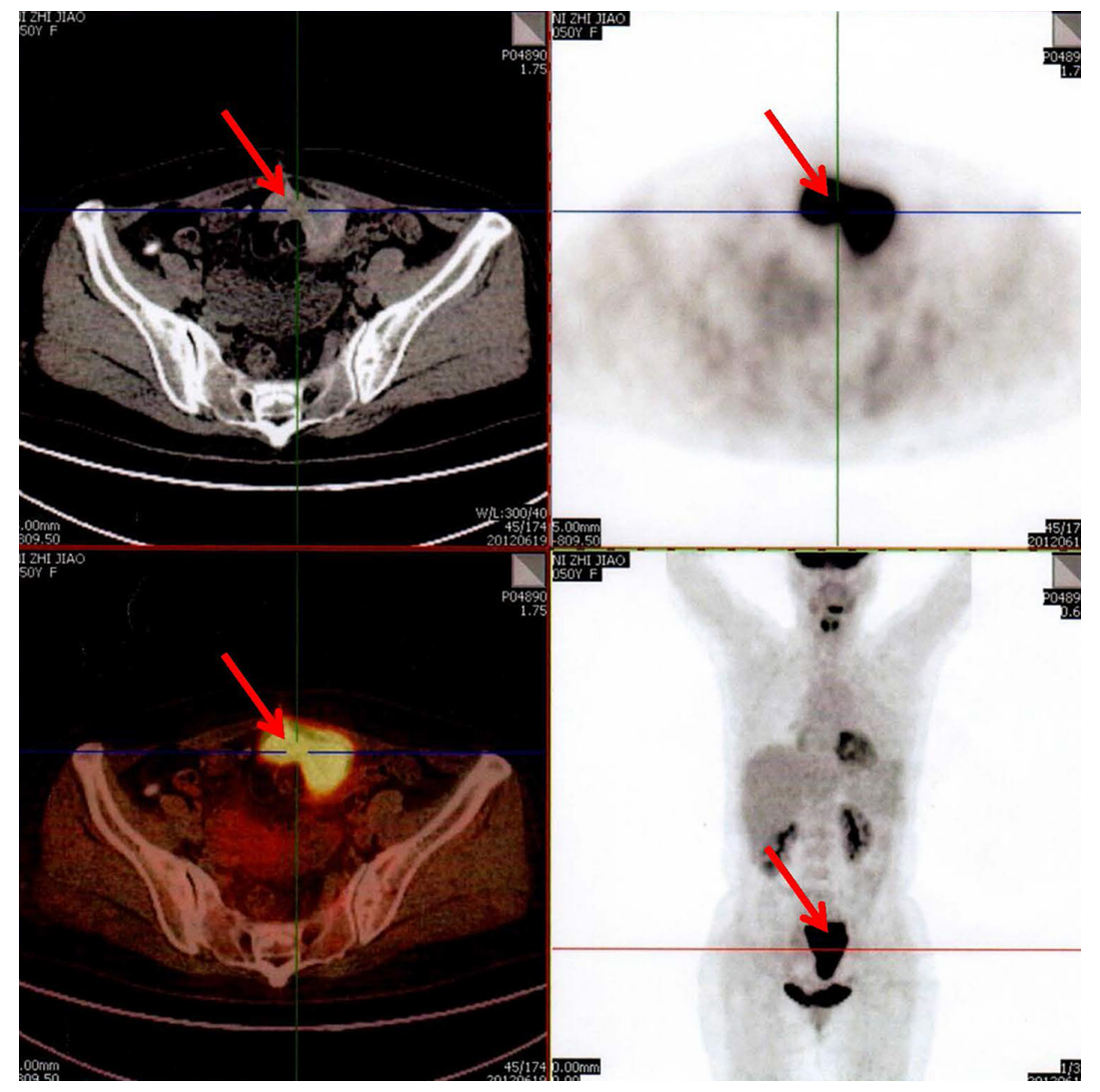

Figure 1 Hypermetabolic mass (red arrows) (SUV ${ }_{\max } 10.7$, delay 1 hour, SUV $\mathrm{max}_{\operatorname{ma}} 14.0$ ) identified on positron emission tomography-CT. 

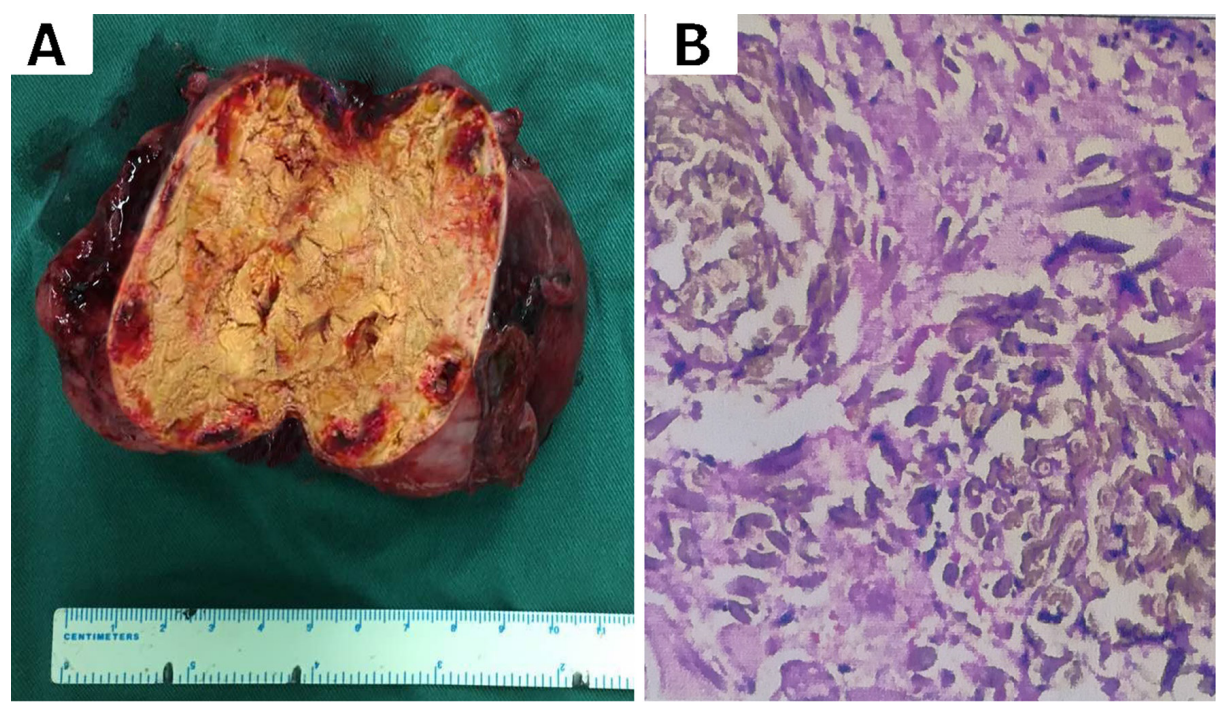

Figure 2 (A) Intraoperative cut surface of the mass demonstrating a retained surgical gauze swab. (B) Histopathological examinations of the surgical gauze swab with features of chronic granuloma.

The current estimate for retained surgical swabs is one event per 10000 procedures. ${ }^{1}$ Gossypiboma, or textiloma, is one of the most commonly retained surgical items. ${ }^{2}$ Although a correct gauze swab count at the end of a procedure may offer some reassurance, a retained surgical gauze swab can still occur. ${ }^{3}$ The highly variable manifestations of gossypiboma often prevent clinicians from making an immediate diagnosis. Although a medical history of previous surgery plays a key role in diagnosis, it was difficult to make an immediate diagnosis in this patient as the high levels of tumour markers in the serum and pleural fluid with a hypermetabolic mass on PET-CT scanning indicated a more serious diagnosis.

Clinicians must be cognisant of the association between retained surgical gauze swabs and pseudo-malignant pleural effusion. Surgical removal and histopathological examination is necessary to confirm the diagnosis.
Contributors All authors managed the patient. TF was responsible for writing, WW and $X G$ for the figure and QG for the literature search.

Funding This work was supported by award from the National Natural Science Foundation of China (nos. 81770095 and 81700093).

Competing interests None declared.

Patient consent Obtained.

Provenance and peer review Not commissioned; externally peer reviewed.

(c) Article author(s) (or their employer(s) unless otherwise stated in the text of the article) 2018. All rights reserved. No commercial use is permitted unless otherwise expressly granted.

\section{REFERENCES}

1 Berger ER, Greenberg CC, Bilimoria KY. Challenges in Reducing Surgical "Never Events". JAMA 2015;314:1386-7.

2 Yeh CC, Kuo YL, Liu HS, et al. Gossypiboma mimicking an ovarian tumor in a young woman with a history of ovarian teratoma. Taiwan J Obstet Gynecol 2010;49:225-7.

3 Possover M. Images in clinical medicine. Gossypiboma in the pouch of Douglas. N Engl J Med 2008:359:e9. 